\title{
Defect-free plastic deformation through dimensionality reduction and self-annihilation of topological defects in crystalline solids
}

\author{
Yipeng Gao $\odot,{ }^{1,2, *}$ Yongfeng Zhang, ${ }^{3}$ Larry K. Aagesen, ${ }^{1}$ Jianguo Yu, ${ }^{1}$ Min Long, ${ }^{4}$ and Yunzhi Wang ${ }^{2}$ \\ ${ }^{1}$ Department of Computational Mechanics and Materials, Idaho National Laboratory, 955 MK Simpson Boulevard, \\ Idaho Falls, Idaho 83415, USA \\ ${ }^{2}$ Department of Materials Science and Engineering, The Ohio State University, Watts Hall, 2041 College Road, Columbus, Ohio 43210, USA \\ ${ }^{3}$ Department of Engineering Physics, University of Wisconsin, 1500 Engineering Drive, Madison, Wisconsin 53706, USA \\ ${ }^{4}$ Department of Computer Science, Boise State University, 1910 University Drive, Boise, Idaho 83725, USA
}

(Received 11 January 2019; revised manuscript received 14 November 2019; accepted 14 January 2020; published 11 February 2020)

\begin{abstract}
As a signature of symmetry-breaking processes, the generation and annihilation of topological defects (domain walls, strings, etc.) are of great interest in condensed matter physics and cosmology. Here we propose a distinctive self-organization process through phase transitions, in which all the generated topological defects are dimensionality reduced and self-annihilated. In crystalline solids, such a unique mechanism allows a perfect single crystal after plastic deformation, which originates from the coupling of different types of broken symmetries.
\end{abstract}

DOI: 10.1103/PhysRevResearch.2.013146

\section{INTRODUCTION}

Topological defects (TDs) are singularities in different dimensions across a wide range of physical systems [1-4]. Despite the fact that the generation of TDs from broken symmetry is well captured through topology [5-8], selfannihilation of TDs during a symmetry-breaking process has been largely overlooked $[9,10]$, especially in complex solid crystal systems where the breaking of different types of symmetries are coupled together. The annihilation of TDs in condensed matter could lead to unique physical properties, e.g., extraordinary electro-optic behaviors in nematic liquid crystal displays and ultrahigh strength in solid single crystals $[11,12]$. In cosmology, the formation, evolution, and elimination of TDs such as domain walls, cosmic strings, and monopoles are crucial to the early history of the universe [5], and the analogs of cosmological phase transitions can be extended to condensed matter systems $[13,14]$. Thus, the self-annihilation of TDs in symmetry-breaking processes could offer some new insights into materials design, which requires detailed understanding of both the topological nature and spatial distribution of defects $[15,16]$. Here we use the phase transition graph (PTG) [17] to investigate the topology and geometry of defects associated with structural phase transitions. In particular, we demonstrate a defect-free deformation of solid crystals through the self-organization of TDs.

\footnotetext{
*Corresponding author: yipeng.gao@inl.gov

Published by the American Physical Society under the terms of the Creative Commons Attribution 4.0 International license. Further distribution of this work must maintain attribution to the author(s) and the published article's title, journal citation, and DOI.
}

For a symmetry-breaking process between two symmetry groups $G \rightarrow H$, the nontrivial topology of the manifold, $M=$ $G / H$ (i.e., coset space of order parameter) dictates the occurrence and dimensionality of TDs $[15,16]$. For example, in solid crystals, a loss of discrete point symmetry during a phase transition produces a nontrivial zeroth-homotopy group of $M$, $\pi_{0}(M)$, which leads to planar defects (e.g., domain boundaries) $[16,18]$. In contrast, a loss of continuous translational symmetry produces a nontrivial first-homotopy group of $M$, $\pi_{1}(M)$, which leads to line defects (e.g., dislocations) $[18,19]$. If the two symmetry-breaking processes are coupled, one may expect special types of defects in terms of dimensionality. We will show a special type of planar domain boundaries generated by phase transitions in crystals. Since the boundaries do not destroy the continuity of crystal lattice, they are essentially reduced to dislocation lines and referred to as topologically reduced domain boundaries (TRDBs). TRDB is a new concept to capture the dimensionality reduction of two-dimensional (2D) topological defects in crystals (e.g., domain boundary).

Meanwhile, in a typical ferroic phase transition, domaindomain interactions can lead to a multidomain selforganization as well as an ordered domain boundary network [20-22]. We will demonstrate that the ordered domain boundary network in some systems vanishes, i.e., domain boundaries are TRDBs that are annihilated in a self-organized manner. In particular, we propose a unique defect-free deformation mechanism through dimensionality reduction and self-annihilation of TRDBs. We choose a typical ferroelastic crystal, $\mathrm{Ni}$ - $\mathrm{Ti}$, and demonstrate that a self-organized "multidomain" structure, dictated by long-range elastic interactions, can be obtained through a biased-load thermal cycling process with all individual domains separated by TRDBs, which are reduced to dislocations being self-annihilated at quadruple junctions. The final deformed material is a perfect single crystal with an adaptivity to respond to various external stress 
(a)
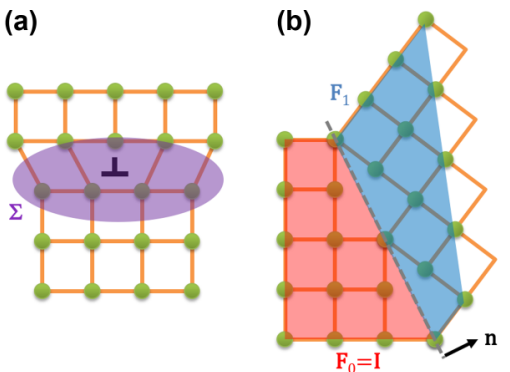

different crystal orientation (c)

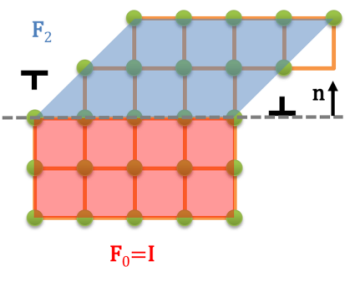

same crystal orientation

FIG. 1. Topological defects in 2D square crystals. (a) A dislocation, (b) a conventional domain boundary, and (c) a special domain boundary reduced to dislocation dipole. ( $\mathbf{F}$ is deformation gradient; $\Sigma$ is the region colored by purple.)

or strain conditions. Since the mechanism could be achieved in any phase transition generating self-organized TRDBs, it suggests a new means of developing superdeformable (superductile) crystals, through repeated macroscopic deformation without accumulating defects.

\section{DIMENSIONALITY REDUCTION OF TOPOLOGICAL DEFECTS IN CRYSTALS}

To fully understand the annihilation mechanism of TDs, we focus on two important phenomena especially associated with TDs in crystals. The first one is dimensionality reduction of domain boundaries. Before a rigorous group theory and topology analysis, the dimensionality reduction phenomenon can be easily understood at an intuitive level, which essentially suggests an intrinsic correlation of defects in different dimensions from a geometric point of view. Three different types of crystalline defects are plotted in the 2D cross section of a simple cubic lattice for simplicity. In Fig. 1(a), a typical dislocation is shown, which is a one-dimensional (1D) defect (dislocation line is normal to the paper plane) originating from the breaking of continuous translational symmetry. In Fig. 1(b), a typical domain boundary is shown, which is a 2D defect (domain boundary is normal to the paper plane) originating from the breaking of mirror symmetry. Note that such a domain boundary could be formed through a deformation process, i.e., the blue domain is deformed (deformation gradient $\mathbf{F}_{1}$ ) relative to the red domain in Fig. 1(b). Since the domain boundary originates from the breaking of mirror symmetry in the simple cubic lattice, it is featured by a specific misorientation of $53.13^{\circ}$ with a specific crystallographic plane, i.e., the (210) plane in the index of the cubic lattice, which is the so-called $\Sigma 5$ twin boundary. Here the notation of $\Sigma 5$ indicates a specific misorientation of $53.13^{\circ}=\sin ^{-1}(0.8)$, which allows atoms from neighboring lattices to coincide, and the density of coinciding sites is $1 / 5$. Figure 1 (c) shows an even special domain boundary, formed through a deformation (deformation gradient $\mathbf{F}_{2}$ ) on the upper blue domain relative to the lower red domain. Even though the dashed line indicates a boundary separating the neighboring domains in different deformation states, it is not a crystallographic planar boundary since it does not destroy lattice continuity. Instead, two dislocation lines (a dipole) are formed. Because the 2D domain

boundary is reduced to 1D dislocation lines, it is essentially a TRDB. Comparing Figs. 1(b) and 1(c), a TRDB can be taken as a special $\Sigma 1$ twin boundary, with no misorientation between neighboring domains. The physical origin of TRDBs, in terms of symmetry breaking, will be further discussed in the following paragraphs.

Meanwhile, domain boundaries and dislocations in crystals can also be taken as singularities in the tensor field of deformation gradient $F_{i j}=\frac{\partial u_{i}}{\partial r_{j}}$ (i.e., derivative of displacement $\left.\mathbf{u}, \mathbf{u}=\mathbf{r}^{\prime}-\mathbf{r}\right)$, constrained by the compatibility condition (i.e., it guarantees a continuous single-valued displacement field) $[20,23]$.

$$
\nabla \times \mathbf{F}(\mathbf{r})=0 .
$$

The compatibility condition has different forms, including closed-path integral and interface jump condition [24,25]. At atomic level, additional information about the discreteness of crystal lattices should be incorporated into the compatibility condition, which generally captures the nature of TDs in crystals $[19,20]$.

$$
\begin{gathered}
\oint_{\partial \Sigma} \mathbf{F}(\mathbf{r}) d \mathbf{l}=\mathbf{b}, \\
\left(\operatorname{cof} \mathbf{F}_{1}-\operatorname{cof} \mathbf{F}_{0}\right) \mathbf{n}=0 .
\end{gathered}
$$

Equation (2) describes a line defect, which is represented by a closed-path integral of $\mathbf{F}(\mathbf{r})$ along the boundary of $\Sigma$ [the purple region in Fig. 1(a)]. The integral value $\mathbf{b}$ is a lattice translation vector, which is called the Burgers vector (of a dislocation) [19]. Note the relation between Eqs. (1) and (2). If it is zero on the right-hand side of Eq. (2), Eq. (2) is the integral form of Eq. (1), which is exactly parallel to the differential and integral forms of the Maxwell equations. Equation (3) describes a planar boundary (with normal vector $\mathbf{n}$ ) between two domains [20]. Inside each domain, the deformation gradient is a constant $\left(\mathbf{F}_{1}\right.$ or $\left.\mathbf{F}_{0}\right)$. cof denotes the cofactor matrix (i.e., cof $\mathbf{F}_{0}=\left|\mathbf{F}_{0}\right| \mathbf{F}_{0}^{-T},\left|\mathbf{F}_{0}\right|$ is the determinant of $\mathbf{F}_{0}$ ). Special domain boundaries are expected when $\mathbf{F}_{1}$ and $\mathbf{F}_{0}$ are related by the symmetry of a crystal lattice. For example, if $\mathbf{F}_{0}$ is the identity $\mathbf{I}$ (undeformed) and $\mathbf{F}_{1}$ is a lattice-invariant deformation of the simple cubic lattice (i.e., $\mathbf{F}_{1}$ transforms a simple cubic lattice to a simple cubic lattice), a twin boundary could be generated [Fig. 1(b)]. In this case, the two domains are different by deformation gradient as well as crystal orientation $\left(53.13^{\circ}\right.$ misorientation). Theoretically, the relation between $\mathbf{F}_{1}$ and $\mathbf{F}_{0}$ could be even special, leading to two neighboring domains with the same crystal orientation. In Fig. 1(c), the blue and red domains are different by deformation gradient $\left(\mathbf{F}_{2}\right.$ vs $\left.\mathbf{F}_{0}\right)$ without a misorientation. As a result, the planar domain boundary does not destroy lattice continuity, which is essentially a TRDB that is reduced to a line defect (a dislocation dipole or a dislocation loop in three dimesions). In this case, a hybrid condition can be established based on Eq. (3) [20],

$$
\mathbf{F}_{2} \mathbf{F}_{0}^{-1}=\mathbf{l}+\mathbf{b} \otimes \mathbf{n},
$$

where $\otimes$ denotes dyadic product, and $\mathbf{n}$ is the boundary plane normal. 
The deformation gradient matrices shown in Fig. 1 are listed as follows:

$$
\begin{aligned}
& \mathbf{F}_{0}=\left[\begin{array}{lll}
1 & 0 & 0 \\
0 & 1 & 0 \\
0 & 0 & 1
\end{array}\right], \\
& \mathbf{F}_{1}=\left[\begin{array}{ccc}
0.6 & -0.2 & 0 \\
0.8 & 1.4 & 0 \\
0 & 0 & 1
\end{array}\right], \\
& \mathbf{F}_{2}=\left[\begin{array}{lll}
1 & 1 & 0 \\
0 & 1 & 0 \\
0 & 0 & 1
\end{array}\right] .
\end{aligned}
$$

By solving $\mathbf{F}_{1} \mathbf{F}_{0}^{-1}=\mathbf{l}+\mathbf{b}_{1} \otimes \mathbf{n}_{1}$, we have

$$
\mathbf{b}_{1}=\left[\begin{array}{c}
-0.2 \\
0.4 \\
0
\end{array}\right], \quad \mathbf{n}_{1}=\left[\begin{array}{l}
2 \\
1 \\
0
\end{array}\right] .
$$

By solving $\mathbf{F}_{2} \mathbf{F}_{0}^{-1}=\mathbf{l}+\mathbf{b}_{2} \otimes \mathbf{n}_{2}$, we have

$$
\mathbf{b}_{2}=\left[\begin{array}{l}
1 \\
0 \\
0
\end{array}\right], \quad \mathbf{n}_{2}=\left[\begin{array}{l}
0 \\
1 \\
0
\end{array}\right] .
$$

It is clear that $\mathbf{b}_{2}$ is a lattice translation vector while $\mathbf{b}_{1}$ is not. As a result, the deformation gradient $\mathbf{F}_{1}$ produces a conventional twin boundary, while $\mathbf{F}_{2}$ produces a TRDB, as shown in Fig. 1. In fact, the TDs shown in Figs. 1(b) and 1(c) correspond to typical crystalline defects in 2D induced by either a deformation on a square lattice or a hexagon to square phase transition $[17,26]$. Note that the two dislocations are exactly opposite to each other (dislocation dipole/loop) in Fig. 1(c), which suggests that the combination of them is topologically trivial. As is well known in materials science, a dislocation loop on its slip plane can easily shrink and self-annihilate. However, during a deformation process in metallic materials, multiple dislocations are generated and entangled, which prevent the annihilation. Similarly, the twin boundary [e.g., in Fig. 1(b)] could also be self-annihilated from a topological point of view, if the twin does not interact with other defects or the external boundary of the material.

Since TRDBs are special types of twin boundaries related to broken symmetry, it is straightforward to expect TRDB generation in a symmetry-breaking process (e.g., structural phase transition). As suggested by previous experimental and theoretical studies [21,27], near-equiatomic Ni-Ti alloys, which undergo a B2 to B19' ferroelastic phase transition, are candidate systems to produce TRDBs through a selforganization process. $\mathrm{B} 2$ is an ordered, bcc-based structure with a space group of $P m \overline{3} m$ (point group $m \overline{3} m$ with 48 symmetry operations), while $\mathrm{B} 19^{\prime}$ is a monoclinic structure with a space group of $P 2_{1} / m$ (point group $2 / m$ with four symmetry operations) [21,28]. Since all the four-point symmetry operations in $\mathrm{B} 19^{\prime}$ originate from $\mathrm{B} 2$, there is a group-subgroup relationship. There are $12(=48 / 4)$ crystallographically equivalent transition pathways [20,21]. According to first-principles calculations $[28,29]$, the B19' structure is geometrically and energetically close to a base-centered orthorhombic structure (BCO; space group $\mathrm{Cmcm}$, point group $\mathrm{mmm}$ with eight symmetry operations), which has a relatively

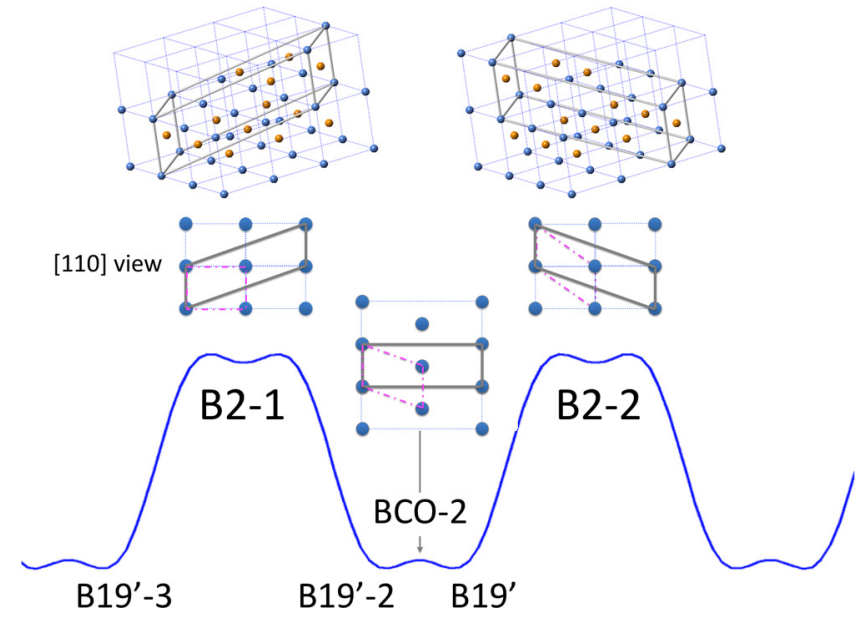

FIG. 2. Schematic drawing of the lattice deformation path and free-energy landscape for the transitions among B2 and B19' states, dictated by the symmetry constraint. The material block delineated by gray lines in B2-1 transform to those blocks delineated by gray lines in BCO-2 and B2-2 (similar for the blocks delineated by magenta lines).

high symmetry in its vicinity (compared to its nearby monoclinic B19' structures). There is a group-subgroup relationship between $\mathrm{BCO}$ and $\mathrm{B}^{\prime}{ }^{\prime}$, and two $(=8 / 4)$ equivalent transition pathways are expected. The structural transitions among B2, $\mathrm{B} 19^{\prime}$, and $\mathrm{BCO}$ are schematically shown in Fig. 2. If the (110) cross section of a B2 structure (B2-1) is considered, $\mathrm{BCO}$ can be formed through a shear of $1 / 2$ [001], and an additional shear of $1 / 2$ [001] will create a new B2 (B2-2) structural state. In Fig. 2, the gray boxes in B2-1 and B2-2 are corresponding atomic cells before and after the lattice deformation. Even though both B2-1 and B2-2 are the same ordered bcc structure, they are related by a lattice-invariant deformation process, and some symmetry operations in B2-1 are lost during the process [17,27]. During such a lattice distortion process, symmetry decreases first and increases again. If we consider the boxes delineated by dash-dotted magenta lines (they are also corresponding to each other), it is clear that B2-1 and B2-2 are symmetric with respect to $\mathrm{BCO}$ (to distinguish this $\mathrm{BCO}$ state from others, we label it $\mathrm{BCO}-2$ ). Since BCO is a high-symmetry structure relative to the nearby B19' states, its energy should correspond to an extreme on the free-energy landscape (a maximum, minimum, or saddle point). The three-dimensional (3D) atomic structures of B2-1 and B2-2 are shown in Fig. 2, while only one set of sublattice atoms (blue atoms) are shown in the $2 \mathrm{D}(110)_{B}$ cross section (the process also involves atomic shuffling). Since B2-1 and B2-2 are related by a mirror symmetry (horizontal mirror) of BCO-2, we usually expect a nontrivial zeroth-homotopy group of the coset space capturing the symmetry-breaking process, which leads to planar defects [15]. However, because the deformation produced by phase transitions coincides with a lattice-invariant deformation of $\mathrm{B} 2$, the effect of mirror symmetry breaking is concealed during the continuous shear deformation, resulting in a trivial zeroth-homotopy group and a nontrivial first-homotopy group. Group theory and topology analyses are presented as follows. 
B2-1
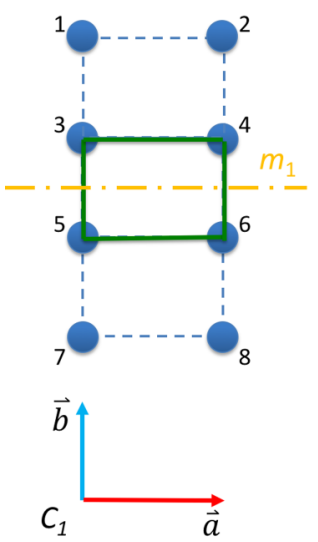

B2-2

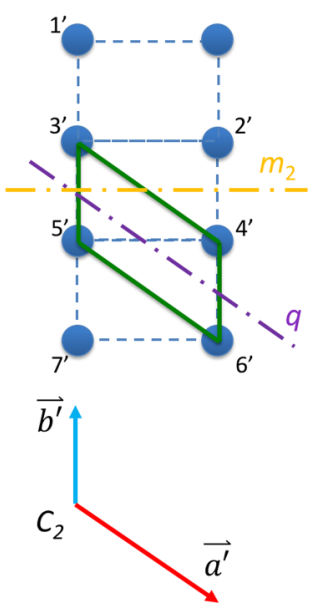

FIG. 3. Crystal structures of and lattice correspondence between B2-1 and B2-2 states ([110] viewing direction).

Here we focus on a transition cycle, during which the initial structural state of B2-1 transforms to B2-2 (Fig. 2). B2-1 and B2-2 are two structural states connected through a lattice distortion (achieved by a phase transition cycling). If we consider B2-1 and B2-2 separately, both of them are in the B2 phase (an ordered bcc phase). However, the symmetry breaking from B2-1 to B2-2 should be determined by the transition from B2-1 and B2-2. Because of the symmetry of a crystal (e.g., point symmetry and translational symmetry), we can deform a crystal into a distorted version of the same lattice, which is the so-called lattice-invariant deformation. During such a lattice distortion process, symmetry decreases first and increases again, which is illustrated in Fig. 3 at an intuitive level. Figure 3 shows the (110) cross section of B2-1 and B2-2 with horizontal mirrors indicated. The lattice distortion is illustrated through the transformation from an original rectangle in the B2-1 state (delineated by green lines) to a parallelogram (delineated by green lines) in B2-2. In this process, each atom in B2-1 transforms to a new position in B2-2. In Fig. 3, eight atoms in B2-1 are illustrated by different numbers (1)-(8). After the transition, atom 1 becomes atom $1^{\prime}$, atom 2 becomes $2^{\prime}$, etc. In other words, there is a one-toone atomic correspondence between B2-1 and B2-2. Such a correspondence can also be described through lattice vector correspondence. As shown in Fig. 3, $\vec{a}$ and $\vec{b}$ vectors (red and blue) in B2-1 are chosen, which correspond to the $\vec{a}^{\prime}$ and $\vec{b}^{\prime}$ vectors in B2-2. The $m_{1}$ mirror in B2-1 reflects atom 3 to 5 , and also reflects atom 4 to 6 . In $\mathrm{B} 2-2$, there is no mirror reflecting atom $3^{\prime}$ to $5^{\prime}$ and reflects atom $4^{\prime}$ to $6^{\prime}$ at the same time, so $m_{1}$ is lost during the transition. For $m_{2}$ in B2-2, it reflects atom $3^{\prime}$ to $5^{\prime}$, and also reflects atom $2^{\prime}$ to $4^{\prime}$. However, there is no mirror reflecting atom 3 to 5 and also reflects atom 2 to 4 in B2-1, so $m_{2}$ is not a symmetry inherited from B2-1. During the transition, the original horizontal mirror plane $m_{1}$ (yellow) transforms to $q$ (purple). Note that $q$ is not a mirror plane in B2-2. The new horizontal mirror plane $m_{2}$ in B2-2 is a new symmetry operation, which is not inherited from B2-1. In summary, during a lattice-invariant deformation process on a
B2 crystal (as indicated by the correspondence between the green rectangle and the parallelogram), the original mirror plane $m_{1}$ disappears and a new mirror plane $m_{2}$ appears. In other words, $m_{1}$ and $m_{2}$ are not corresponding to each other during the transition.

The broken symmetry associated with the transition from B2-1 to B2-2 can also be determined rigorously using group action description. A crystal lattice in $n$ dimension can be fully described by $n$ linear independent lattice vectors. One $n \times n$ matrix, $\mathbf{A}$, can be constructed by using the $n$ column lattice vectors (i.e., each lattice vector becomes a column of the matrix), which fully captures the crystal lattice. Note that this is not a unique description, because of the symmetry of the lattice. Theoretically, the same lattice can be described by another matrix B (also formed by column lattice vectors):

$$
\mathbf{B}=\mathbf{A} \cdot q_{\mathrm{AB}}, \quad q \in G L(n, Z) .
$$

Here $G L(n, Z)$ is the general linear group in $n$ dimension over integers $(Z)$. The general linear group $G L(n, Z)$ is the set of $n \times n$ invertible matrices, with all the entries of the matrices being integers. In other words, all the bases describing the same lattice can be related through a number of transformations (e.g., $q$ ), which form a group, $G L(n, Z)$. $G L(n, Z)$ is a generalized symmetry group, which describes the self-automorphism of a crystal lattice.

We consider the metric of $\mathbf{A}, \mathbf{A}^{T} \mathbf{A}$. All the metrics of lattice vector matrices, which describe the same lattice (as $\mathbf{A}^{T} \mathbf{A}$ describes), can be included in a set $X_{A}$.

$$
X_{A}=\left\{q^{T} \mathbf{A}^{T} \mathbf{A} q, q \in G L(n, Z)\right\} .
$$

Here a group $G L(n, Z)$ acts on $X$ (the metric for all $n$ dimensional lattice vector sets), through a group action in the form of $q^{T} x q . X_{A}$ is a subset of $X$, which is the orbit of $\mathbf{A}^{T} \mathbf{A}$. In other words, if $\mathbf{B}$ and $\mathbf{A}$ are related by Eq. (5), $\mathbf{A}^{T} \mathbf{A}$ and $\mathbf{B}^{T} \mathbf{B}$ are in the same orbit $X_{A}$, which also describe the same type of lattice.

The so-called lattice group associated with $\mathbf{A}, \mathbf{P}_{\mathrm{A}}$, is defined as the stabilizer subgroup of $G L(n, Z)$ with respect to $\mathbf{A}^{T} \mathbf{A}$.

$$
\mathbf{P}_{\mathrm{A}}=\left\{q: q^{T} \mathbf{A}^{T} \mathbf{A} q=\mathbf{A}^{T} \mathbf{A}, q \in G L(n, Z)\right\} .
$$

$\mathbf{P}_{\mathrm{A}}$ can be considered as a specific matrix representation of the point group of the $\mathbf{A}$ lattice.

Similarly, the lattice group associated with $\mathbf{B}, \mathbf{P}_{\mathrm{B}}$, can be obtained.

$$
\mathbf{P}_{\mathrm{B}}=\left\{q: q^{T} \mathbf{B}^{T} \mathbf{B} q=\mathbf{B}^{T} \mathbf{B}, q \in G L(n, Z)\right\} .
$$

Considering the relation between $\mathbf{B}$ and $\mathbf{A}$ through Eq. (5), we can find that $\mathbf{P}_{\mathrm{A}}$ and $\mathbf{P}_{\mathrm{B}}$ are conjugated subgroups.

$$
\mathbf{P}_{\mathrm{B}}=q_{\mathrm{AB}}^{-1} \mathbf{P}_{\mathrm{A}} q_{\mathrm{AB}} .
$$

In order to determine the symmetry breaking associated with a transition from $\mathbf{A}$ to $\mathbf{B}$ [e.g., Eq. (5)], we consider another group action, which is different from Eq. (6). We define a group action of $\mathbf{P}_{\mathrm{A}}$ on $G L(n, Z)$, in the form of $p^{-1} q p, q \in$ $G L(n, Z)$. Note that $\mathbf{P}_{\mathrm{A}}$ is a subgroup of $G L(n, Z)$.

The stabilizer subgroup of $\mathbf{P}_{\mathrm{A}}$ with respect to $q_{\mathrm{AB}}$ can be obtained.

$$
\mathbf{P}_{\mathrm{AB}}=\left\{p: p^{-1} q_{\mathrm{AB}} p=q_{\mathrm{AB}}, p \in \mathbf{P}_{\mathrm{A}}\right\} .
$$


Since $\mathbf{P}_{\mathrm{A}}$ and $\mathbf{P}_{\mathrm{B}}$ are conjugated subgroups of $G L(n, Z)$, related through $q_{\mathrm{AB}}$ in Eq. (9), $\mathbf{P}_{\mathrm{AB}}$ includes the element in $\mathbf{P}_{\mathrm{A}}$ that does not change by the conjugacy (i.e., commute with $\left.q_{\mathrm{AB}}\right)$. As a result, $\mathbf{P}_{\mathrm{AB}}$ includes all the elements that are common for $\mathbf{P}_{\mathrm{A}}$ and $\mathbf{P}_{\mathrm{B}}$, and it can be proved that $\mathbf{P}_{\mathrm{AB}}=\mathbf{P}_{\mathrm{A}} \cap \mathbf{P}_{\mathrm{B}}$. The stabilizer $\mathbf{P}_{\mathrm{AB}}$ includes the preserved symmetry elements during the transition from $\mathbf{A}$ to $\mathbf{B}$. As a result, the symmetry elements in $\mathbf{P}_{\mathrm{A}}$ but not in $\mathbf{P}_{\mathrm{AB}}$ are the lost symmetry.

The above general method can be utilized to determine the broken symmetry associated with the transition from B2-1 to B2-2. In Fig. 3, it is clear that the transition can be reduced to $2 \mathrm{D}$, since there is no change along the third dimension (i.e., [110] axis in B2). As a result, $\vec{a}$ and $\vec{b}$ vectors describe B2-1, while $\overrightarrow{a^{\prime}}$ and $\overrightarrow{b^{\prime}}$ vectors describe B2-2. We choose those vectors in the way that they are corresponding vectors in the transition (i.e., $\vec{a}$ corresponds to $\overrightarrow{a^{\prime}}, \vec{b}$ corresponds to $\vec{b}^{\prime}$ ). For convenience, we choose a coordinate system $C_{1}$ (formed by $\vec{a}$ and $\vec{b}$ vectors). The matrices for B2-1 and B2-2 (with column lattice vectors) can be determined as

$$
\mathbf{A}_{\mathrm{B} 2-1}=\left[\begin{array}{ll}
1 & 0 \\
0 & 1
\end{array}\right], \quad \mathbf{A}_{\mathrm{B} 2-2}=\left[\begin{array}{cc}
1 & 0 \\
-1 & 1
\end{array}\right] .
$$

According to Eq. (7), the $m_{1}$ mirror symmetry can be determined as follows, which transforms $\vec{a} \rightarrow \vec{a}$ and $\vec{b} \rightarrow-\vec{b}$.

$$
m_{1} \rightarrow\left[\begin{array}{cc}
1 & 0 \\
0 & -1
\end{array}\right]
$$

Similarly, the $m_{2}$ mirror symmetry can be determined, which transforms $\overrightarrow{a^{\prime}} \rightarrow \overrightarrow{a^{\prime}}+2 \overrightarrow{b^{\prime}}$ and $\overrightarrow{b^{\prime}} \rightarrow-\overrightarrow{b^{\prime}}$.

$$
m_{2} \rightarrow\left[\begin{array}{cc}
1 & 0 \\
2 & -1
\end{array}\right]
$$

It is clear that $m_{1}$ and $m_{2}$ are two different mirror symmetry operations, respectively, which do not correspond to each other. In other words, during the transition from B2-1 to B2-2, the original mirror $m_{1}$ disappears, while a new mirror $m_{2}$ appears.

In fact, a number of original symmetry operations in B21 disappear during such a symmetry-breaking process. For simplicity, we only consider the loss of this mirror symmetry $m_{1}$. The symmetry group for $m_{1}$ mirror symmetry can be determined.

$$
P_{1}=\left\{\left[\begin{array}{ll}
1 & 0 \\
0 & 1
\end{array}\right],\left[\begin{array}{rr}
1 & 0 \\
0 & -1
\end{array}\right]\right\} .
$$

In addition, continuous symmetry originating from the translational symmetry of continuum can be described as

$$
T_{c}=\left\{\left[\begin{array}{ll}
1 & 0 \\
y & 1
\end{array}\right], y \in R\right\} .
$$

Geometrically, $T_{c}$ is the continuous group including all simple shear deformations with a vertical shear vector, which is isomorphic to the one-dimensional translational group, $T_{c} \cong$ $T$ (1). Similarly, we have a discrete symmetry group capturing the translational symmetry of a crystal lattice,

$$
T_{d}=\left\{\left[\begin{array}{ll}
1 & 0 \\
n & 1
\end{array}\right], n \in Z\right\} .
$$

It is clear that $T_{d}$ is isomorphic to an infinite cyclic group, $T_{d} \cong Z . T_{d}$ is a subgroup of $T_{c}$, and dislocations (line defects) originate from the symmetry breaking from $T_{c}$ to $T_{d}$, which is not necessarily related to a phase transition. The homotopy groups of $T_{c} / T_{d}$ are $[15,16]$

$$
\begin{aligned}
& \pi_{0}\left(T_{c} / T_{d}\right)=0, \\
& \pi_{1}\left(T_{c} / T_{d}\right)=Z,
\end{aligned}
$$

which suggest the line defects caused by the loss of continuous translational symmetry, but no planar defects.

Here we consider the coupling between the losses of discrete point symmetry (i.e., horizontal mirror $m_{1}$ ) and continuous translational symmetry, which is originally included in a group $G$. $G$ is a group including both translational symmetry and mirror symmetry,

$$
G=\left\{\left[\begin{array}{cc}
1 & 0 \\
y & \pm 1
\end{array}\right], y \in R\right\} .
$$

If the product state (e.g., a rotated version of B2-2 relative to B2-1) after the transition only has translational symmetry inherited from the parent state (e.g., B2-1), it can be described by $T_{d}$, and defect structures during the symmetry-breaking process (from $G$ to $T_{d}$ ) are dictated by the homotopy groups of $G / T_{d}$,

$$
\begin{aligned}
\pi_{0}\left(G / T_{d}\right) & =2, \\
\pi_{1}\left(G / T_{d}, x\right) & =Z,
\end{aligned}
$$

which suggest the existence of both planar defects and line defects.

Here we consider B2-2 (the same orientation with B2-1) as the product state in 2D (Fig. 3), with its own horizontal mirror symmetry operation $m_{2}$. The symmetry group for $m_{2}$ mirror symmetry can be

$$
P_{2}=\left\{\left[\begin{array}{ll}
1 & 0 \\
0 & 1
\end{array}\right],\left[\begin{array}{cc}
1 & 0 \\
2 & -1
\end{array}\right]\right\} .
$$

Here we include both the point symmetry and translational symmetry of the product state B2-2 through group $H$, which is generated by the following generating set:

$$
S_{H}=\left\{\left[\begin{array}{cc}
1 & 0 \\
2 & -1
\end{array}\right],\left[\begin{array}{ll}
1 & 0 \\
n & 1
\end{array}\right]\right\}, \quad n \in Z .
$$

In fact, we can find

$$
H=\left\{\left[\begin{array}{cc}
1 & 0 \\
n & \pm 1
\end{array}\right], n \in Z\right\} \cong 2 \times Z .
$$

Note the difference between $H$ and $T_{d} . T_{d}$ does not include a mirror symmetry because the mirror symmetry in rotated B2-2 is not inherited from B2-1 during the transition. However, 
mirror symmetry exists in $H$ because $m_{2}$ in nonrotated B22 corresponds to a compound symmetry operation, i.e., the combination of $m_{1}$ and translational symmetry. At an intuitive level, such a phenomenon requires the same crystal orientation between B2-1 and B2-2 domains [similar to the red and blue domains in Fig. 1(c)], which is essentially linked through a lattice-invariant and orientation-invariant deformation.

The topological defects generated by the symmetrybreaking process from $G$ to $H$ are dictated by the homotopy groups of $G / H$,

$$
\begin{aligned}
& \pi_{0}(G / H)=0, \\
& \pi_{1}(G / H)=Z,
\end{aligned}
$$

which suggest that there are only line defects but no planar defect.

From the above analysis, we know that the loss of discrete symmetry (mirror symmetry in the above example) may not necessarily generate planar defects. For a certain transition path, planar defects are reduced to line defects because of the coupling of mirror symmetry and translational symmetry. Mirror symmetry is critical in determining the zeroth-homotopy group. The rotational symmetry of B2 is not considered for simplicity, which is not directly related to this specific type of topologically reduced domain boundary.

In mathematics, the dimensionality reduction of TDs is similar to the description of Green's theorem, in which the contribution from a 2D surface is reduced to that from its boundary. In a discrete fcc lattice, it is well known that an additional shear on a Shockley partial dislocation can result in a perfect dislocation. Here a Shockley partial (planar defect) is similar to a $\mathrm{B} 2 / \mathrm{BCO}$ interface, while a perfect dislocation (line defect) is similar to the topologically reduced domain boundary between B2-1 and B2-2. As a matter of fact, the deformation gradients of B2-1 and B2-2 satisfy the condition that produces TRDBs, i.e., Eq. (4), which results from the coupling of different types of broken symmetry. The topological dimensionality reduction of defects is especially important for the construction of the final defect-free crystal, in which all the "planar boundaries" are reduced to "dislocation lines" and then self-annihilated.

\section{SELF-ORGANIZATION OF TOPOLOGICAL DEFECTS IN CRYSTALS}

In addition to dimensionality reduction, the annihilation of TDs also requires a self-organization process. Note that TRDBs could have the properties of both domain boundary and dislocation. On the one hand, domain-domain interaction could provide an intrinsic driving force for self-organization, which results in an ordered spatial alignment of domain boundaries. On the other hand, the dimensionality-reduced TDs in crystals (i.e., dislocations) could interact and react with each other, and dislocations with opposite Burgers vectors could neutralize and disappear.

To achieve a multidomain self-organization, we need several new B2 states to form different domains. According to the symmetry breaking associated with the $\mathrm{B} 2$ to $\mathrm{B} 19^{\prime}$ transition, 12 B19' states (B19'-2-B19'-13) can be generated by a one-way phase transition, so that 12 new B2 states (B2-2-B2-13) can be generated by a transition cycle. As observed in experiments [30,31], four B19' states form a herringbonelike multidomain structure, which is periodically repeated to satisfy the compatibility for bulk materials. Such a unique multidomain structure is the result of self-organization dominated by long-range elastic interactions during phase transitions, which has been widely observed in Ni-Ti-based alloys [20,21]. To precisely select the four transition pathways and domain states that lead to self-organized TRDBs, we employ a PTG approach. PTG is a newly developed tool to analyze the crystalline defects generated by symmetry associated with phase transitions, which has been successfully utilized in steels [32,33], Ti alloys [34], and a number of ferroic smart materials $[17,35]$. In a PTG, each vertex represents a structural state, while each edge between two vertices represents a pathway connecting two states [17]. According to symmetry relations along the pathways, each B2 state is connected with $12 \mathrm{~B} 19^{\prime}$ states, which constructs a local star structure (with one B2 and twelve B19' states). Each BCO state is connected with two B19' states, and each B19' state is connected with one B2 state and one BCO state. In Fig. 4, nine local stars are interconnected with twelve $\mathrm{BCO}$ states as bridges. The multiedge path connecting B2-1/B19'-2/BCO2/B19'/B2-2 in Fig. 4 corresponds to the lattice distortion sequence in Fig. 2. Details on the construction of PTG in Ni-Ti can be found in the literature $[17,35]$. To make the transition to the new B2-2-B2-5 states energetically favorable, an external load, e.g., a biaxial tension along $[010]_{\mathrm{B} 2}$ and $[001]_{\mathrm{B} 2}$ directions, can be applied to bias the transition pathway. Such a biased-load favors the B2-2-B2-5 and B19'-2-B19'-5 states, which is critical to make all TRDBs self-annihilable. Without precisely designed biased-load, nonannihilable TRDBs (i.e., dislocations) are widely observed in experiments $[21,27]$.

A defect-free deformation process is shown in Fig. 5 (domain structure) and Fig. 6 (atomic structure), i.e., a single crystal in the B2-1 state transforms to a herringbonelike structure formed by four B19' domains (B19'-2, B19'-3, B19'-4, and $\mathrm{B} 19^{\prime}-5$ domains are colored by light-blue, green, yellow, and red, respectively, in Fig. 5). Then each B19' domain transforms to a new B2, e.g., B19'-2 transforms to B2-2. Similar to the analyses of TRDB between B2-1 and B2-2 in Eq. (4), it can be demonstrated that all the domain boundaries (i.e., between B2-2 and B2-3, between B2-3 and B2-4, between B24 and B2-5, and between B2-5 and B2-1) in the final deformed crystal are TRDBs, with all the dislocations at the quadruple junctions annihilated (Fig. 6). The final structure is a perfect crystal free of defects, which can be repeated periodically in bulk materials. Note the difference between the initial and final perfect crystals. The final crystal is obtained by a nonuniform deformation of the initial one, during which each individual domain undergoes a lattice-invariant deformation. The atomic structure of the defect-free deformation process is shown in Fig. 6, generated by using OvITO software [36]. Atoms in the two sublattices of B2 are colored by purple and white, while those of $\mathrm{B} 19^{\prime}$ are colored by purple and blue/green/yellow/red (the same color scheme as the domain state shown in Fig. 5). In the right column of Fig. 6, it is clear that the final deformed B2 crystal is a perfect single crystal. 


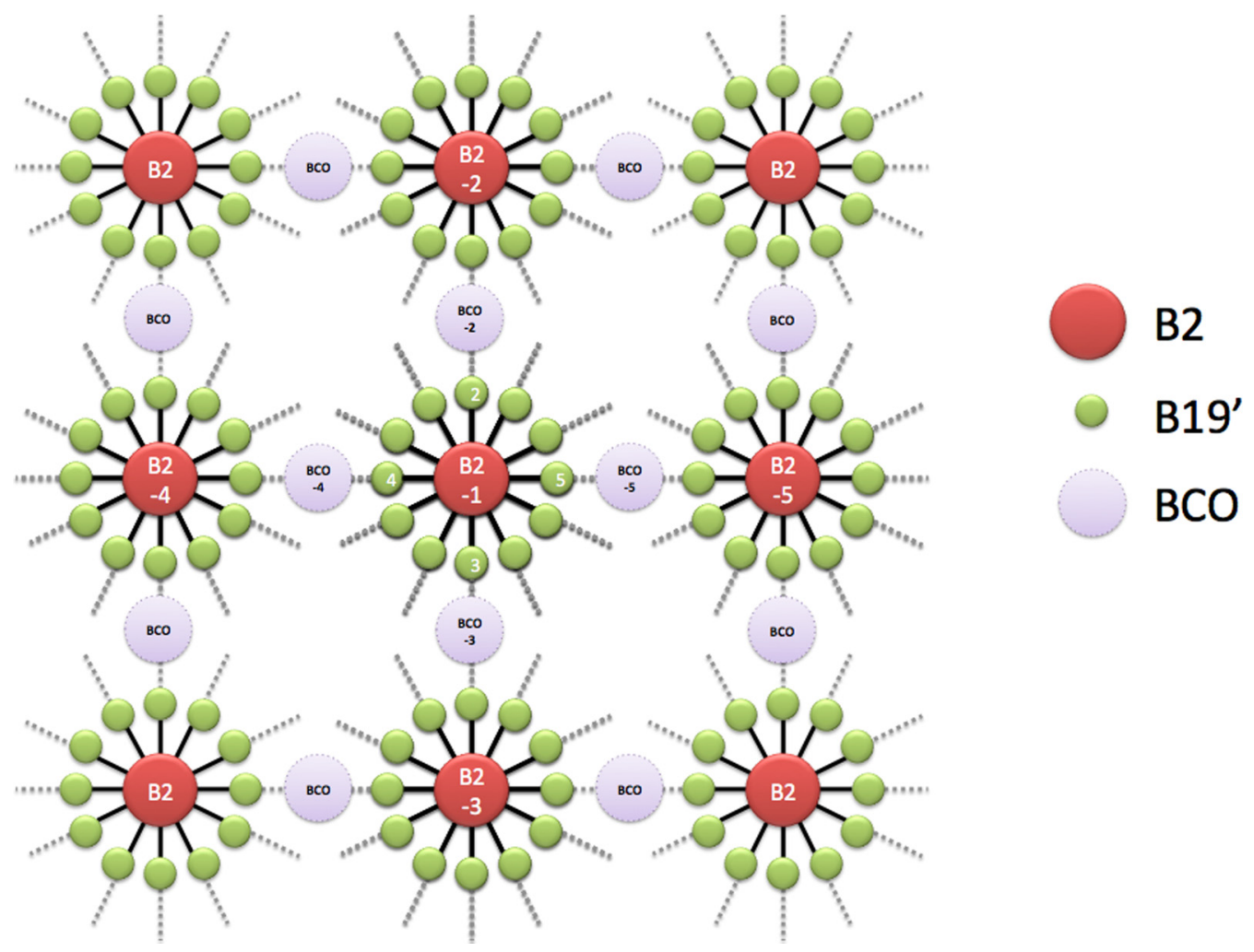

FIG. 4. Phase transition graph for the B2-B19' transition in Ni-Ti alloys. The phase transition pathway between B2 and B19' states are described by solid lines, while the non-phase-transition pathways between B19' states (through BCO) are described by dashed lines.

The deformation gradient matrices for B2-2, B2-3, B2-4, and B2-5 are listed as follows [37].

$$
\begin{array}{ll}
\mathbf{F}_{2}=\left[\begin{array}{ccc}
1 & 0.5 & -0.5 \\
0 & 1 & 0 \\
0 & 0 & 1
\end{array}\right], & \mathbf{F}_{3}=\left[\begin{array}{ccc}
1 & -0.5 & 0.5 \\
0 & 1 & 0 \\
0 & 0 & 1
\end{array}\right], \\
\mathbf{F}_{4}=\left[\begin{array}{ccc}
1 & 0.5 & 0.5 \\
0 & 1 & 0 \\
0 & 0 & 1
\end{array}\right], & \mathbf{F}_{5}=\left[\begin{array}{ccc}
1 & -0.5 & -0.5 \\
0 & 1 & 0 \\
0 & 0 & 1
\end{array}\right] .
\end{array}
$$

All the boundaries in the herringbonelike structure can be theoretically determined through phase transition crystallog- raphy [38-40]. The mathematical conditions for the formation of such a self-organized structure are as follows [20]:

$$
\begin{aligned}
& \mathbf{Q}_{32} \mathbf{F}_{3}-\mathbf{F}_{2}=\mathbf{b}_{32} \otimes \mathbf{n}_{32}, \\
& \mathbf{Q}_{43} \mathbf{F}_{4}-\mathbf{F}_{3}=\mathbf{b}_{43} \otimes \mathbf{n}_{43}, \\
& \mathbf{Q}_{54} \mathbf{F}_{5}-\mathbf{F}_{4}=\mathbf{b}_{54} \otimes \mathbf{n}_{54}, \\
& \mathbf{Q}_{25} \mathbf{F}_{2}-\mathbf{F}_{5}=\mathbf{b}_{25} \otimes \mathbf{n}_{25}, \\
& \mathbf{n}_{32}, \mathbf{n}_{43}, \mathbf{n}_{54}, \mathbf{n}_{25} \text { lie on a plane. }
\end{aligned}
$$

It can be proved that Eq. (23) is another form of the interface jump condition, Eq. (2) [20]. In the above equations, the only inputs are the deformation gradient matrices $\left(\mathbf{F}_{2}-\mathbf{F}_{5}\right)$. high temperature

B2 single crystal

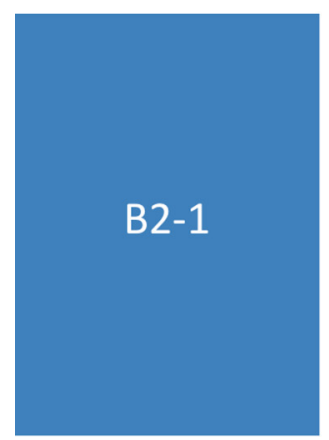

low temperature

B19' multi-domain

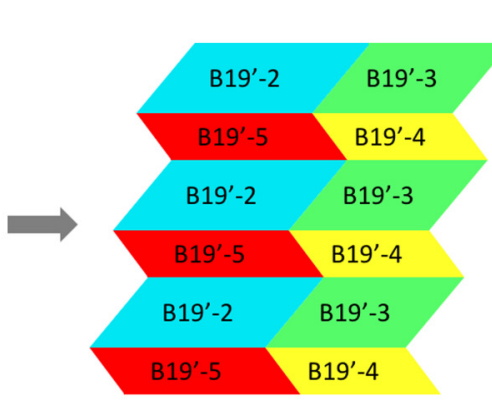

high temperature

B2 single crystal (deformed)

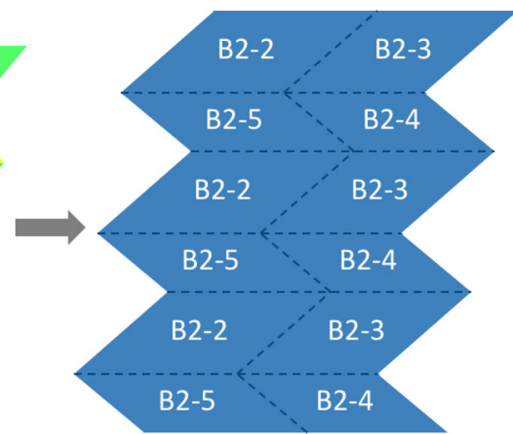

FIG. 5. Schematic drawing of a defect-free deformation process through a biased-load thermal cycling in a Ni-Ti alloy: an undeformed B2 crystal transforms to a B19' multidomain structure, and then transforms to a deformed B2 crystal without defects. The dashed lines in B2 states indicate the topologically reduced domain boundaries. 


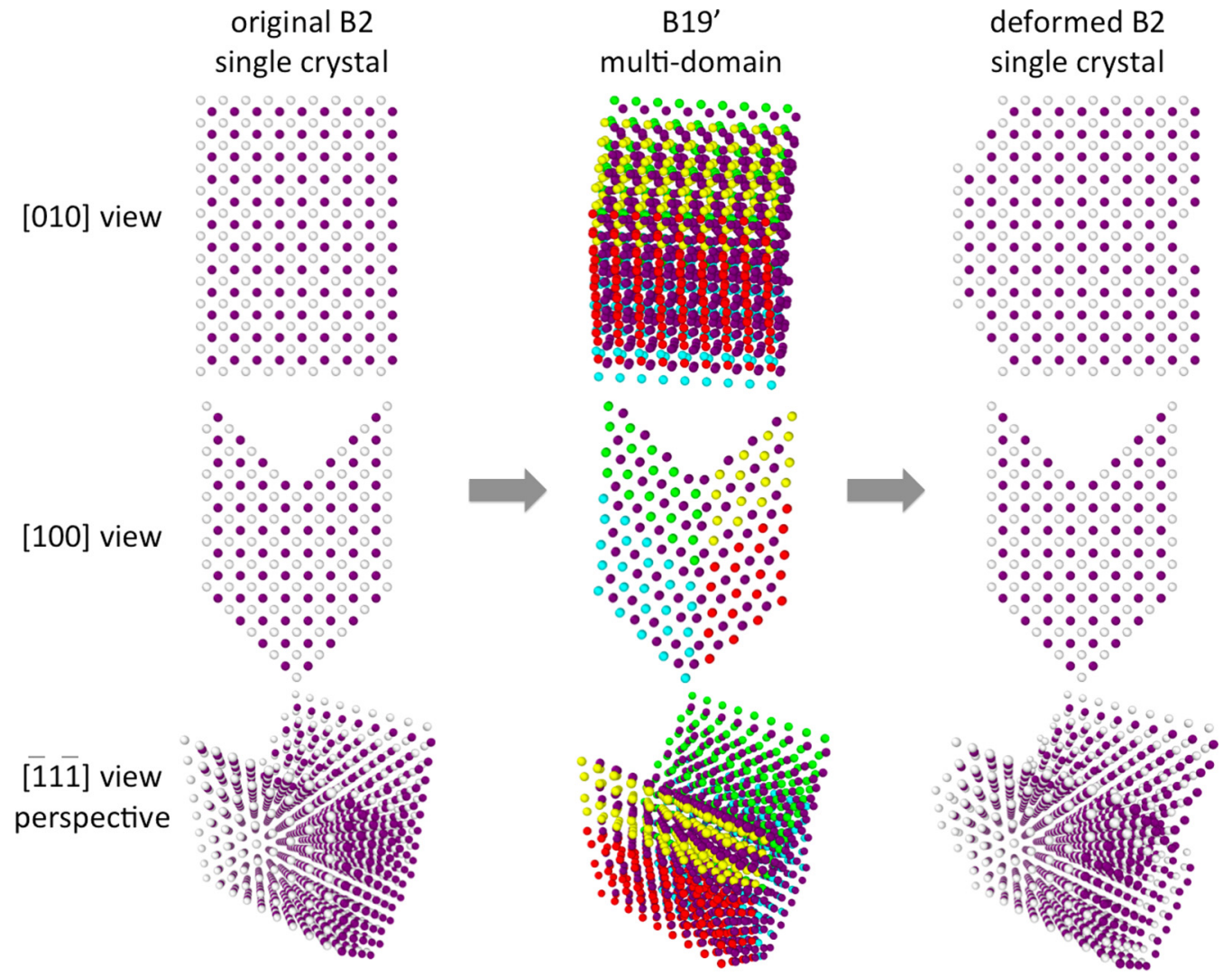

FIG. 6. Atomic structure of the defect-free deformation process through biased-load thermal cycling: an undeformed B2 crystal transforms to a B19' multidomain structure, and then transforms to a deformed B2 crystal without defects.

And the domain boundary planes as well as misorientation can be determined through $\mathbf{n}$ (boundary plane normal) and $\mathbf{Q}$ (rotation matrix).

$$
\begin{aligned}
& \mathbf{b}_{32}=\left[\begin{array}{l}
1 \\
0 \\
0
\end{array}\right], \quad \mathbf{n}_{32}=\left[\begin{array}{r}
0 \\
-1 \\
1
\end{array}\right], \quad \mathbf{Q}_{32}=\left[\begin{array}{lll}
1 & 0 & 0 \\
0 & 1 & 0 \\
0 & 0 & 1
\end{array}\right] \\
& \mathbf{b}_{43}=\left[\begin{array}{r}
-1 \\
0 \\
0
\end{array}\right], \quad \mathbf{n}_{43}=\left[\begin{array}{r}
0 \\
-1 \\
0
\end{array}\right], \quad \mathbf{Q}_{43}=\left[\begin{array}{lll}
1 & 0 & 0 \\
0 & 1 & 0 \\
0 & 0 & 1
\end{array}\right] \\
& \mathbf{b}_{54}=\left[\begin{array}{r}
-1 \\
0 \\
0
\end{array}\right], \quad \mathbf{n}_{54}=\left[\begin{array}{l}
0 \\
1 \\
1
\end{array}\right], \quad \mathbf{Q}_{54}=\left[\begin{array}{lll}
1 & 0 & 0 \\
0 & 1 & 0 \\
0 & 0 & 1
\end{array}\right] \\
& \mathbf{b}_{25}=\left[\begin{array}{l}
1 \\
0 \\
0
\end{array}\right], \quad \mathbf{n}_{25}=\left[\begin{array}{l}
0 \\
1 \\
0
\end{array}\right], \quad \mathbf{Q}_{25}=\left[\begin{array}{lll}
1 & 0 & 0 \\
0 & 1 & 0 \\
0 & 0 & 1
\end{array}\right] .
\end{aligned}
$$

The types of domain boundaries can be determined through $\mathbf{n}$ and $\mathbf{Q}$ obtained above (in the crystallographic index of B2-1).

According to the topological analysis in the previous section, all the 2D "domain boundaries" are reduced to 1D dislocations (or dislocaiton loops), i.e., all the Q's are the identity matrix (zero misorientation). Note the dislocation lines at the quadruple junctions. In Fig. 7(a), a dislocation loop (reduced from a domain boundary) between B2-2 and B2-5 is illustrated. According to Eq. (24), its shear vector (i.e., Burger vector) is [100], with the line direction (i.e., sense vector in dislocation theory) determined by the plane normal through the right-hand rule in Fig. 7(a). To make it self-consistent, all the plane normals are properly chosen so that the Burgers vector can be directly summed together at the quatriple junction. As a result, it can be easily proved that all the shear vectors ( $\mathbf{b}_{32}, \mathbf{b}_{43}, \mathbf{b}_{54}$, and $\mathbf{b}_{25}$, ) are summed to zero, which essentially suggests a defect-free structure.

In order to precisely select the structural states of B2-2-B25 , we need an external bias to make those states energetically favorable. Since the B2 to B19' transition in Ni-Ti is a typical

(a)

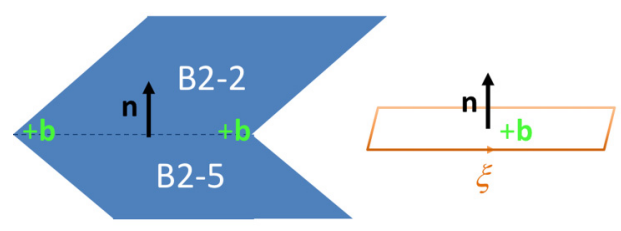

(b)

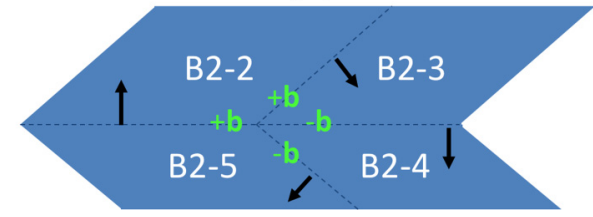

FIG. 7. (a) Dislocations generated between B2-2 and B2-5 domains, with Burgers vector and sense vector indicated. (b) Selfannihiliation of dislocations at a quatriple junction. 
phase transition in reponse to stress field, we expect that a biased stress could serve the purpose. The eigenstrains for the structural states of B2-2-B2-5 can be determined through a Lagrangian finite strain formula,

$$
\begin{gathered}
\mathbf{e}_{i}=\frac{\mathbf{F}_{i}^{T} \mathbf{F}_{i}-\mathbf{I}}{2}, \\
\mathbf{e}_{2}=\left[\begin{array}{ccc}
0 & 0.25 & -0.25 \\
0.25 & 0.125 & -0.125 \\
-0.25 & -0.125 & 0.125
\end{array}\right], \\
\mathbf{e}_{3}=\left[\begin{array}{ccc}
0 & -0.25 & 0.25 \\
-0.25 & 0.125 & -0.125 \\
0.25 & -0.125 & 0.125
\end{array}\right] \\
\mathbf{e}_{4}=\left[\begin{array}{ccc}
0 & 0.25 & 0.25 \\
0.25 & 0.125 & 0.125 \\
0.25 & 0.125 & 0.125
\end{array}\right], \\
\mathbf{e}_{5}=\left[\begin{array}{ccc}
0 & -0.25 & -0.25 \\
-0.25 & 0.125 & 0.125 \\
-0.25 & 0.125 & 0.125
\end{array}\right] .
\end{gathered}
$$

From the above eigenstrains, it is clear that a biaxial tensile stress (along the $[010]_{\mathrm{B} 2}$ and $[001]_{\mathrm{B} 2}$ directions) is the best biased-load condition to make exactly the four B2 states (i.e., B2-2-B2-5) energetically favorable.

In the ideal case, the final crystal is free of internal stress, and the volume fractions of the four kinds of domains follow $f_{\mathrm{B} 2-2}: f_{\mathrm{B} 2-3}=f_{\mathrm{B} 2-5}: f_{\mathrm{B} 2-4}$, which meets the compatibility condition of Eq. (3) with all line defects self-annihilated. Plastic deformation is carried by domains. Unless the volume fractions of the four kinds of domains are equal (25\% each), there is a macroscopic shape change after the whole process. The shape change does not produce any crystalline defect after the transition cycle, which can adapt to various external stress or strain conditions by adjusting domain volume fractions. The self-organization of TRDBs is dictated by the dynamics of phase transitions. As suggested by previous experimental and theoretical studies [20,21,30,31,41,42], the herringbonelike modulated B19' structure (Fig. 5) is formed through autocatalysis and self-accommodation, dominated by long-range elastic interactions among multiple domains. The characteristic length of the modulated structure, i.e., domain size, is determined by the interplay of domain boundary energy $\left(\gamma: J / m^{2}\right)$ and elastic energy $\left(\lambda \varepsilon^{2}: J / m^{3}\right)$ [43],

$$
l \simeq \gamma /\left(\lambda \varepsilon^{2}\right),
$$

where $\lambda$ is the elastic modulus and $\varepsilon$ is the eigenstrain strain of the B2 to B19' transition. As suggested by Eq. (27), a low domain boundary energy (comparing to elastic energy) leads to a fine multidomain structure. Since all domain boundaries are compatible, such a multidomain B19' structure is thermodynamically stable, as indicated by experimental observations [30,31]. Upon the B19' to B2 transition, the multidomain B19' structure can transform either back to the original B2 crystal or to the deformed one (Fig. 5), both of which are perfect crystals. However, the external stress biases the choice of transition pathways, leading to the deformed crystal. As reported in previous experimental and theoretical studies [44,45], domains and dislocations can be generated by transition cycling in a Ni-Ti system at a uniaxial stress of 100-200 MPa [27,37]. To make the domain formation more favorable than non-self-annihilable dislocations, we suggest a low level of biased load during the B2 to B19' transition upon cooling $(\sim 50 \mathrm{MPa})$, which assists the pathway selection but does not induce non-self-annihilable dislocation [17,42]. During the B19' to B2 transition upon heating, a relatively high level of biased load is recommended to drive the domains to new structural states rather than the original $(\sim 150 \mathrm{MPa})$. The biased-load stress should always be lower than the yield strength of either the B2 or the B19' phase. In this paper, we only show the transition cycling caused by temperature change. Mechanical cycling may also activate such a defectfree deformation process through stress-induced phase transition. Because the initial state is deformed to final state without involving any defect (i.e., both are perfect crystals), repeat deformation would suggest a new means to design superdeformable "playdough" metals and alloys.

\section{CONCLUSION}

In this paper, two characteristic phenomena associated with crystalline defects, dimensionality reduction and selforganization, have been investigated through group theory, graph theory, and topology. It has been found that the combination of those two phenomena lead to a unique defectfree deformation mechanism in Ni-Ti alloys, which originates from the coupling of different types of broken symmetries. Such a unique mechanism not only suggests a new way to develop superdeformable metals and alloys, but also provides new insight to understand the generation, evolution, and annihilation of topological defects.

\section{ACKNOWLEDGMENTS}

Y.G. and Y.W. acknowledge the financial support of U.S. Department of Energy under Grant No. DE-SC0001258. Y.G., L.K.A., and J.Y. acknowledge the support of U.S. Department of Energy under DOE-NE Idaho Operations Office Contract No. DE-AC07-05ID14517.
[1] P. Bhattacharjee, C. T. Hill, and D. N. Schramm, Phys. Rev. Lett. 69, 567 (1992).

[2] T. C. Lubensky, D. Pettey, N. Currier, and H. Stark, Phys. Rev. E 57, 610 (1998).

[3] I. Muševič, M. Škarabot, U. Tkalec, M. Ravnik, and S. Žumer, Science 313, 954 (2006).
[4] O. V. Yazyev and S. G. Louie, Phys. Rev. B 81, 195420 (2010).

[5] T. W. B. Kibble, J. Phys. A: Math. Gen. 9, 1387 (1976).

[6] G. Toulouse and M. Kléman, J. Phys. Lett. 37, 149 (1976).

[7] R. Shankar, J. Phys. 38, 1405 (1977).

[8] V. Poenaru and G. Toulouse, J. Phys. 38, 887 (1977). 
[9] L. Giomi, M. J. Bowick, X. Ma, and M. C. Marchetti, Phys. Rev. Lett. 110, 228101 (2013).

[10] R. R. Guimarães, R. S. Mendes, P. R. G. Fernandes, and H. Mukai, J. Phys.: Condens. Matter 25, 404203 (2013).

[11] S. W. Choi, Y. Takanishi, K. Ishikawa, and H. Takezoe, Appl. Phys. Lett. 90, 033115 (2007).

[12] K. E. Petersen, Proc. IEEE 70, 420 (1982).

[13] W. H. Zurek, Nature (London) 317, 505 (1996).

[14] W. H. Zurek, U. Dorner, and P. Zoller, Phys. Rev. Lett. 95, 105701 (2005).

[15] N. D. Mermin, Rev. Mod. Phys. 51, 591 (1979).

[16] H. R. Trebin, Adv. Phys. 31, 195 (1982).

[17] Y. Gao, S. A. Dregia, and Y. Wang, Acta Mater. 127, 438 (2017).

[18] M. Kléman, L. Michel, and G. Toulouse, J. Phys. Lett. 38, 195 (1977).

[19] J. M. Burgers, Proc. Phys. Soc. 52, 23 (1940).

[20] K. Bhattacharya, Microstructure of Martensite (Oxford University Press, New York, 2004).

[21] K. Otsuka and X. Ren, Prog. Mater. Sci. 50, 511 (2005).

[22] C. Kittel, Rev. Mod. Phys. 21, 541 (1949).

[23] J. Lubliner, Plasticity Theory (Dover Publications, New York, 2008).

[24] A. Yavari, Arch. Ration. Mech. Anal. 209, 237 (2013).

[25] J. Casey, Int. J. Struct. Change Solids 3, 61 (2011).

[26] R. Baggio, E. Arbib, P. Biscari, S. Conti, L. Truskinovsky, G. Zanzotto, and O. U. Salman, Phys. Rev. Lett. 123, 205501 (2019).

[27] M. L. Bowers, Y. Gao, L. Yang, D. J. Gaydosh, M. De Graef, R. D. Noebe, Y. Wang, and M. J. Mills, Acta Mater. 91, 318 (2015).
[28] X. Huang, G. J. Ackland, and K. M. Rabe, Nat. Mater. 2, 307 (2003).

[29] W. S. Ko, Scr. Mater. 154, 134 (2018).

[30] M. Nishida, K. Yamauchi, I. Itai, H. Ohgi, and A. Chiba, Acta Metall. Mater. 43, 1229 (1995).

[31] M. Nishida, H. Ohgi, I. Itai, A. Chiba, and K. Yamauchi, Acta Metall. Mater. 43, 1219 (1995).

[32] K. Bhattacharya, S. Conti, G. Zanzotto, and J. Zimmer, Nature (London) 428, 55 (2004).

[33] Y. Gao and Y. Wang, Phys. Rev. Mater. 2, 093611 (2018).

[34] Y. Gao, Y. Zhang, B. W. Beeler, and Y. Wang, Phys. Rev. Mater. 2, 073402 (2018).

[35] Y. Gao, Materialia 6, 100320 (2019).

[36] A. Stukowski, Model. Simul. Mater. Sci. Eng. 18, 015012 (2010).

[37] Y. Gao, L. Casalena, M. L. Bowers, R. D. Noebe, M. J. Mills, and Y. Wang, Acta Mater. 126, 389 (2017).

[38] C. M. Wayman, Introduction to the Crystallography of Martensitic Transformation (Collier-Macmillan, New York, 1964).

[39] J. S. Bowles and J. K. Mackenzie. Acta Metall. 2, 129 (1954).

[40] M. S. Wechsler, D. S. Lieberman, and T. A. Read, Trans. AIME 197, 1503 (1953).

[41] G. B. Olson and M. Cohen, Ann. Rev. Mater. Sci. 11, 1 (1981).

[42] Y. Gao, N. Zhou, D. Wang, and Y. Wang, Acta Mater. 68, 93 (2014).

[43] A. G. Khachaturyan, Theory of Structural Transformations in Solids (Wiley, New York, 1983).

[44] S. Rajagopalan, A. L. Little, M. A. M. Bourke, and R. Vaidyanathan, Appl. Phys. Lett. 86, 081901 (2005).

[45] P. Chowdhury and H. Sehitoglu, Prog. Mater. Sci. 85, 1 (2017). 